\title{
Hiperparatiroidismo Primário na Pós-menopausa
}

\author{
Primary Hyperparathyroidism after Menopause \\ Martha Katherine Paniagua Huayllas, Dolores Perovano Pardini, \\ Aníbal Tagliaferri, Ana Maria Menezes \\ RESUMO
}

\begin{abstract}
A osteoporose é uma doença que acomete milhões de pacientes no mundo inteiro, levando a complicações muitas vezes graves e até ao óbito. A prevenção e o diagnóstico precoce se fazem necessários para o sucesso da terapêutica, porém existem doenças que podem cursar concomitantemente. O hiperparatiroidismo primário é um diagnóstico que deve ser lembrado em mulheres na pós-menopausa.
\end{abstract}

PALAVRAS-CHAVE: Menopausa. Hiperparatiroidismo. Osteoporose.

\section{Introdução}

A osteoporose na pós-menopausa é a doença osteometabólica com maior prevalência no mundo ocidental e resulta de uma perda de massa óssea superior a 30\% em 10 a 20 anos após a falência ovariana, refletindo a importância do esteróide sexual na manutenção da homeostase óssea na menacme, a qual pode ser demonstrada pela eficácia da terapia de reposição hormonal (TRH) ${ }^{1}$.

O hiperparatiroidismo primário (HPP) consiste em uma alteração do metabolismo mineral causada por secreção excessiva de paratormônio (PTH) a partir das glândulas paratiróides geralmente adenomatosas ou hiperplásicas, podendo resultar em hipercalcemia discreta, moderada ou gra$\mathrm{ve}^{2}$. Atualmente o HPP é considerado uma doença comum e isto se deve à inclusão da dosagem sérica de cálcio nos exames de rotina, o que facilita o diagnóstico precoce ${ }^{3}$. Cerca de 100.000 novos casos são diagnosticados a cada ano, sendo as mulheres duas vezes mais afetadas que os homens $^{2}$.

A maioria dos pacientes não evidenciam sinais ou sintomas de doença óssea, renal, gastrointestinal ou neuromuscular e constituem

Centro de Saúde e Disciplina de Endocrinologia do Departamento de Medicina da Escola Paulista de Medicina - UNIFESP

Correspondência: Martha Katherine P. Huayllas

Disciplina de Endocrinologia

Universidade Federal de São Paulo - UNIFESP

Caixa Postal 20.266

04034-970 - São Paulo - SP - Fax (11) 570-0095

e-mail: marthaka@uol.com.br o grupo com HPP assintomático. No Brasil, porém, o diagnóstico ainda é feito tardiamente, o que aumenta a proporção de casos sintomáticos graves. Isto ocorre provavelmente devido à falta de inclusão das dosagens de cálcio sérico na rotina $^{2}$. A maioria dos pacientes com HPP têm diminuição do osso cortical com relativa preservação do osso trabecular.

Reduzida massa cortical óssea observada principalmente nos ossos dos braços e pernas é uma indicação aceitável para cirurgia ${ }^{4}$. O maior desafio quanto à conduta desta doença é a decisão em relação a quais pacientes deveriam se submeter à paratiroidectomia, a única opção atual de cura.

Nós relatamos o caso de uma paciente menopausada com piora na densitometria de massa óssea na vigência de TRH. A investigação complementar revelou HPP devido a um adenoma benigno de paratiróide.

O diagnóstico de HPP deve ser lembrado nessa população de pacientes, uma vez que as faixas etárias das duas patologias se sobrepõem e a melhora da osteoporose pode ocorrer após a cirurgia.

\section{Relato do Caso}

P.C., 66 anos, branca, brasileira, menopausa fisiológica aos 43 anos. Procurou o Serviço de Climatério do Centro de Saúde da Escola Paulista de Medicina para avaliação de TRH iniciada em outro serviço há 1 ano. Estava em uso de estrógenos conjugados $(0,625 \mathrm{mg} / \mathrm{dia})$ e medroxiprogestero- 
na $(5 \mathrm{mg} /$ dia por 12 dias/mês) regularmente. Referia fogachos, irritabilidade e dores ósseas. Relatava atividade física leve e baixa ingestão alimentar de cálcio (menor que $500 \mathrm{mg} /$ dia). Nos exames de rotina a paciente apresentava lipides, glicemia, mamografia e ultra-sonografia pélvica normais. A densitometria óssea inicial analisada pelo densitômetro DPX lunar revelou osteoporose importante (Figura 1).

Foi realizada investigação complementar para avaliação de marcadores bioquímicos do metabolismo do cálcio (Tabela 1). Chamavam a atenção os niveis elevados de paratormônio PTH basal: 199 pg/ml (normal 10 a 70) e de cálcio sérico 11,5 $\mathrm{mg} / \mathrm{dl}$ (normal 8,5 a 10,5). A ultra-sonografia cervical revelou uma imagem hiperecogênica, sólida, heterogênea, de contornos regulares, medindo cerca de $2,5 \mathrm{~cm}$, situada em topografia do lobo tiroideano esquerdo.
A paciente foi submetida a paratiroidectomia esquerda, cujo exame anátomo-patológico revelou a presença de adenoma benigno.

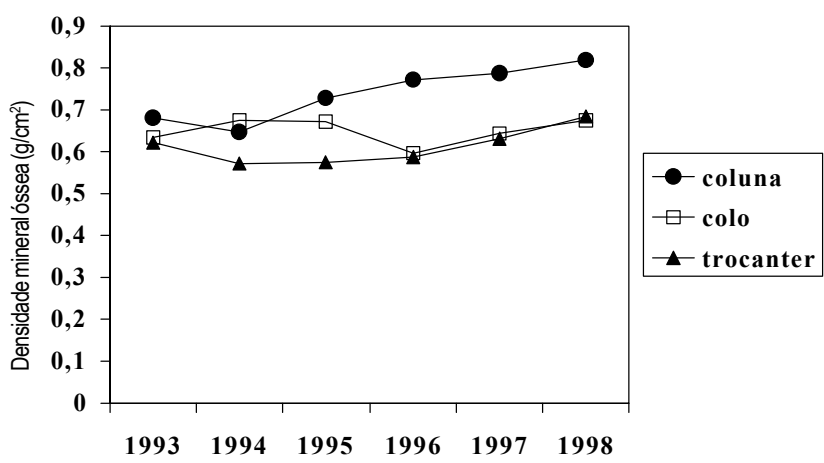

Figura 1 - Valores anuais da densitometria óssea antes e após a ressecção do adenoma de paratireóide.

Tabela 1 - Marcadores bioquímicos do metabolismo ósseo antes e após a cirurgia.

\begin{tabular}{|c|c|c|c|c|c|c|c|c|c|}
\hline & $\begin{array}{l}\text { PTH } \\
\text { pg/dl }\end{array}$ & $\begin{array}{c}\mathrm{Ca} \\
\mathrm{mg} / \mathrm{dl}\end{array}$ & $\begin{array}{c}P \\
\mathrm{mg} / \mathrm{dl}\end{array}$ & $\begin{array}{c}\text { Ca/ion } \\
\text { mg/dl }\end{array}$ & $\begin{array}{r}\text { F A } \\
\text { U/I }\end{array}$ & $\begin{array}{c}\mathrm{Ca} \mathrm{U} \\
\mathrm{mg} / 24 \mathrm{hs}\end{array}$ & $\begin{array}{l}\text { Pyr } \\
\text { pg/umo } \\
\text { Creat }\end{array}$ & $\begin{array}{l}\text { D Pyr } \\
\text { pg/umo } \\
\text { Creat }\end{array}$ & $\begin{array}{l}\mathrm{TSH} \\
\mu \mathrm{U} / \mathrm{ml}\end{array}$ \\
\hline Pré-op & 199 & 11,5 & 2,0 & 1,37 & 103 & 856 & 53 & 8 & ND \\
\hline Pós- op & 49 & 8,6 & 2,6 & 1,21 & 214 & 112 & ND & ND & 1,0 \\
\hline
\end{tabular}

PTH (paratormônio), Ca (cálcio), P (fósforo), Ca/ion (cálcio ionizável), FA (fosfatase alcalina), Ca U (cálcio urinário), Pyr ( pridinolina), D Pyr (deoxipiridinolina), TSH (hormônio tirotrófico). ND, Não determinado.

\section{Discussão}

O hiperparatiroidismo primário (HPP) é uma doença endócrina que, muitas vezes se apresenta como hipercalcemia assintomática ${ }^{5,6}$.

Em muitos pacientes não se encontram os critérios para o tratamento cirúrgico sugerido pelo Consenso para Conduta no HPP Assintomático ${ }^{7} \mathrm{e}$ aproximadamente metade é submetida a cirurgia. Tais critérios são: a) cálcio sérico maior que $12 \mathrm{mg} / \mathrm{dl}$, b) calciúria maior que $400 \mathrm{mg} / \mathrm{g}$ de creatinina/dia, c) manifestações de HPP como: nefrolitíase, osteite fibrosa cística, doença neuromuscular, d) marcada redução na densidade mineral óssea cortical ( $Z$ score $<2,5 \mathrm{DP})$, e) clearance de creatinina reduzido na ausência de outra causa, f) idade inferior a 50 anos.

A decisão para se optar por cirurgia no HPP assintomático não é fácil. A resposta definitiva para esta questão depende da progressão da doença e da análise conjunta dos critérios ${ }^{8}$.

Sabe-se que o PTH tem duplo efeito no esqueleto: catabólico no osso cortical e anabólico no trabecular ${ }^{4}$, e nos pacientes com HPP típico as lesões ocorrem em sítios de osso cortical com preservação relativa do osso trabecular, como por exemplo o rádio e a coluna.

Porém, em um subgrupo de pacientes, observou-se que a densidade mineral óssea da coluna lombar estava muito abaixo dos valores normais quando o diagnóstico de HPP era feito, sendo este achado uma nova indicação para cirurgia, uma vez que havia melhora na densitometria óssea após o tratamento cirúrgico desses pacientes ${ }^{4}$.

Este é um outro aspecto que deve ser comentado. Existe pouca informação disponível sobre a melhora ou reversibilidade do envolvimento ósseo após a paratiroidectomia ${ }^{9}$. Dados antigos da literatura descrevem dramática remineralização óssea em pacientes com osteíte fibrosa cística, porém, isto não se aplica atualmente, pois é difícil o encontro de tais lesões.

A necessidade de enfocar o acometimento do osso trabecular é de particular relevância na mulher menopausada, que constitui o grande grupo de pacientes que, acometido pela deprivação estrogênica, tem maiores riscos de fratura de coluna e fêmur. 
A paciente descrita vinha apresentando piora progressiva da densitometria óssea, principalmente no fêmur, e só houve recuperação na massa óssea após a cirurgia, tendo ocorrido um ganho de $14 \%$ na coluna lombar, $8 \%$ no colo do fêmur, $8 \%$ no triângulo de Ward e $15 \%$ no trocanter.

O diagnóstico de HPP deve ser lembrado nesta população de pacientes uma vez que as faixas etárias de ambas as patologias se sobrepõem, e a dosagem dos marcadores do metabolismo ósseo, principalmente cálcio sérico, deve ser incluida na rotina.

\section{ABSTRACT}

Osteoporosis is an important disease which can affect millions of patients all over the world, leading to complications, often even to death. Prevention and the early diagnosis may help in the success of treatment but there are diseases which can occur at the same time. Primary hyperparathyroidism is a diagnosis which must be remembered in women after the menopause.

KEY WORDS: Osteoporosis. Hyperparathyroidism. Menopause.

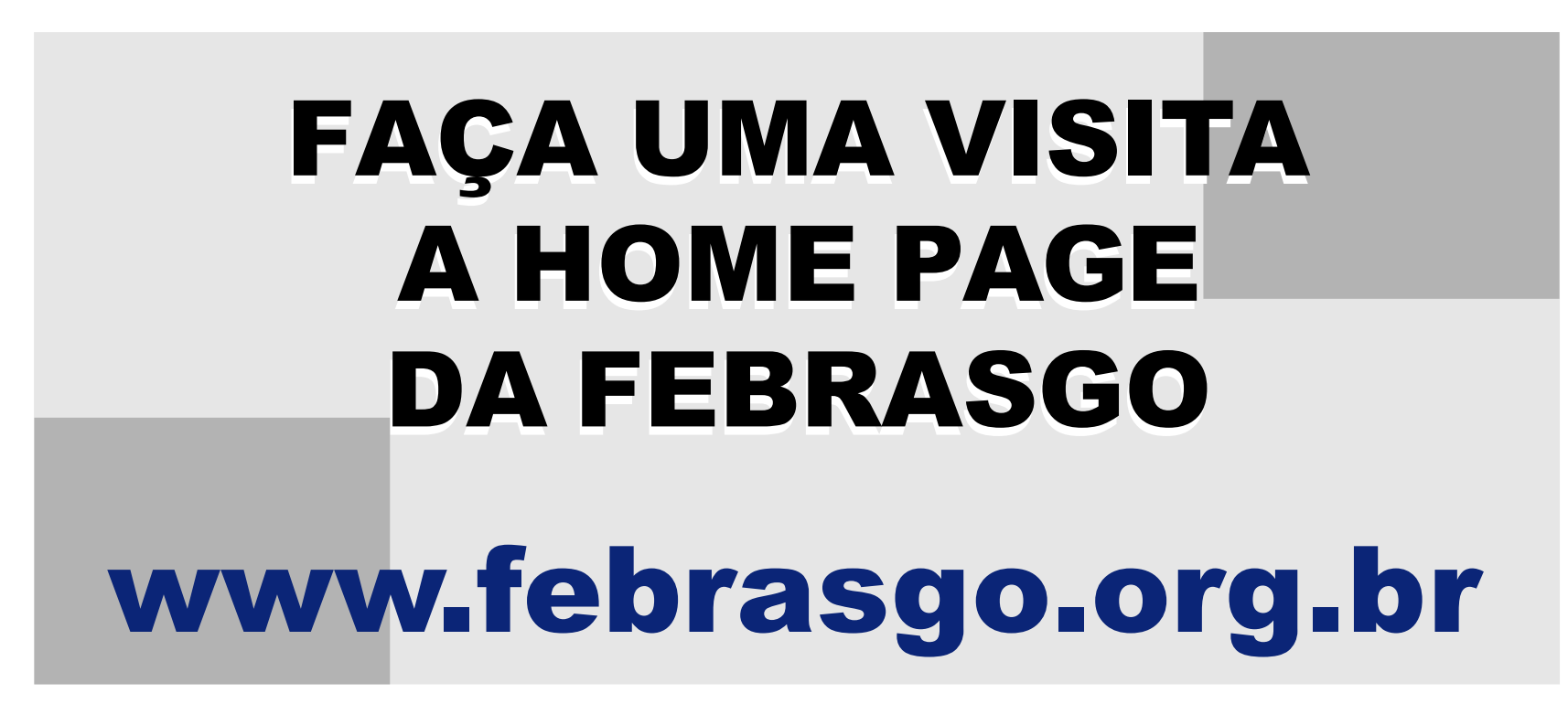

\section{Referências}

1. Hughes DE, Boyce BF. Estrogen, transforming growth factor beta, and the regulation of bone metabolism in health and disease. Endocrinologist 1998; 8:55-61.

2. Hauache OM, Amarante ECJ, Mendoza B, et al. Análise clínica, laboratorial e achados cirúrgicos de 28 casos de hiperparatiroidismo primário. Arq Bras Endocrinol Metab 1995; 39:157-62.

3. al Zahrani A, Levine MA. Primary hyperparathyrodism. Lancet 1997; 349:1233-8.

4. Silverberg SJ, Locker F, Bilezikian JP. Vertebral osteopenia: a new indication for surgery in primary hyperparathyroidism. J Clin Endocrinol Metab 1996; 81:4007-12.

5. Heath H 3d, Hodgson SF, Kennedy M. Primary hyperparathyroidism. Incidence morbidity and potential economic impact in a community. $\mathrm{N}$ Engl J Med 1980; 302:189-93.

6. Scholz DA, Purnell DC. Asymptomatic primary hyperparathyroidism. 10-year prospective study. Mayo Clin Proc 1981; 56:473-8.

7. Consensus development conference statement on primary hyperparathyroidism. J Bone Miner Res 1991; 6 Suppl 2:9-13.

8. Silverberg SJ, Gartenberg F, Jacobs TP, et al. biochemical indices in untreated primary hyperparathyroidism. J Clin Endocrinol Metab 1995; 80:723-8.

9. Silverberg SJ, Gartenberg F, Jacobs TP, et al. Increased bone mineral density after parathyroidectomy in primary hyperparathyroidism. J Clin Endocrinol Metab 1995; 80:729-34. Longitudinal measurements of bone density and 